\title{
Pedicled posterior pericardial repair of tracheoesophageal fistula after chemoradiotherapy for esophageal cancer
}

\author{
Takushi Yasuda, MD, ${ }^{\mathrm{a}}$ Tomoki Makino, MD, ${ }^{\mathrm{b}}$ Osamu Shiraishi, MD, ${ }^{\mathrm{a}}$ and Shunsuke Sogabe, MD, ${ }^{\mathrm{a}}$ \\ Osaka, Japan
}

\footnotetext{
From the ${ }^{\mathrm{a}}$ Department of Surgery, Faculty of Medicine, Kinki University, and ${ }^{\mathrm{b}}$ Department of Gastroenterological Surgery, Graduate School of Medicine, Osaka University, Osaka, Japan. Disclosures: Authors have nothing to disclose with regard to commercial support.

Received for publication Nov 30, 2015; revisions received Jan 15, 2016; accepted for publication Jan 20, 2016 available ahead of print March 3, 2016.

Address for reprints: Takushi Yasuda, MD, Department of Surgery, Faculty of Medicine, Kinki University, 377-2 Ohno-Higashi, Osaka-Sayama, Osaka 589-8511, Japan (E-mail: tyasuda@surg.med.kindai.ac.jp).

J Thorac Cardiovasc Surg 2016;151:e95-7

$0022-5223 / \$ 36.00$

Copyright (C) 2016 by The American Association for Thoracic Surgery

http://dx.doi.org/10.1016/j.jtcvs.2016.01.045
}

Tracheoesophageal fistula is one of the life-threatening adverse events associated with definitive chemoradiotherapy (DCRT). ${ }^{1}$ However, severe scarring caused by DCRT renders a surgical approach especially difficult, and a tailored advanced technique was required to save the patient's life. Here we report a case of tracheoesophageal fistula after DCRT that was successfully treated by patch closure with a pedicled posterior pericardial flap.

\section{CLINICAL SUMMARY}

A 66-year-old male patient was diagnosed simultaneously with a 5-cm superficial circumferential esophageal squamous cell carcinoma (ESCC) of stage IA (T1aNOM0) and dermatomyositis at another hospital. He underwent 60-Gy DCRT using cisplatin and 5-fluorouracil for the ESCC and high-dose steroid therapy for the dermatomyositis. ESCC disappeared completely after DCRT, but 4.5 months later, the patient was rushed to the hospital because of sudden severe coughing and high-grade fever. Upper gastrointestinal endoscopy demonstrated circumferential necrosis affecting three-fourths of the esophageal wall (Figure 1,A), as well as fistula formation opening into the left main bronchus (LMB), approximately $1 \mathrm{~cm}$ in diameter. Two days later, he was transferred to our hospital. At this point, the esophageal balloon of the Sengstaken-Blakemore tube placed to occlude the fistula had blocked the lumen of the LMB almost completely (Figure 1, $B$ ) and enlarged the fistula, which extended from the trachea to the LMB (Figure 1, $C$ ). Considering the large size of the fistula and the near-circumferential necrosis of the esophageal wall that would likely develop into an aortoesophageal fistula, we determined that thoracic esophagectomy and repair of the airway defect were needed to save the patient's life.

Three days after hospital admission, right thoracotomy was performed under percutaneous cardiopulmonary support using venoatrial extracorporeal membrane oxygenation (ECMO) via the femoral access site along with artificial ventilation with a double-lumen endotracheal tube placed in the LMB distal to the fistula. Careful separation of the esophagus from the aorta in the area with severe

\section{DISCUSSION}

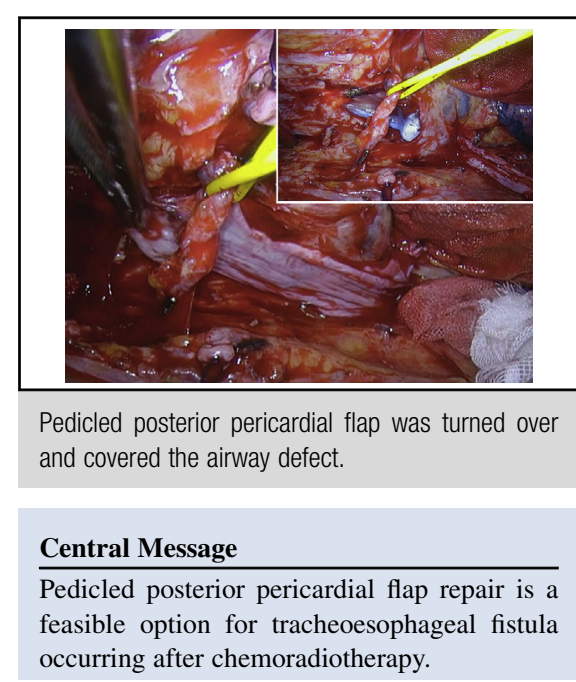

See Editorial Commentary page e99.

scarring revealed that the necrotic esophageal wall had almost disappeared and the aortic wall was directly exposed to the esophageal lumen. Subsequently, the esophagus was meticulously separated from the tracheobronchial tree, revealing a defect measuring $4 \times 2.5 \mathrm{~cm}$ (Figure $2, A$ ).

After thoracic esophagectomy, a posterior pericardial flap was harvested via a downward incision of the posterior pericardium in a horseshoe shape at the base of the oblique pericardial sinus. Then the airway defect was closed with the flap turned up using absorbable polydioxanone 4-0 interrupted sutures (PDS II; Ethicon, Somerville, NJ) (Figure 2, $B$ and $C$ ). Finally, a pedicled right latissimus dorsi muscle flap was used to reinforce the patch repair. Surgery was completed without esophageal reconstruction, given that saving the patient's life was more urgent and because of his long-term, high-dose steroid therapy, preoperative poor nutritional status, and excessive surgical stress.

The postoperative course was uneventful (Figure 2,D). Pathological examination revealed no tumor cells in the resected specimen. Esophageal reconstruction was performed successfully with a gastric tube thorough the subcutaneous route in a 2 -stage procedure 2.5 months later. No fistula or tumor recurrence was detected at 10 months postsurgery.

Fistula formation between the esophagus and the tracheobronchial airway is a life-threatening complication 

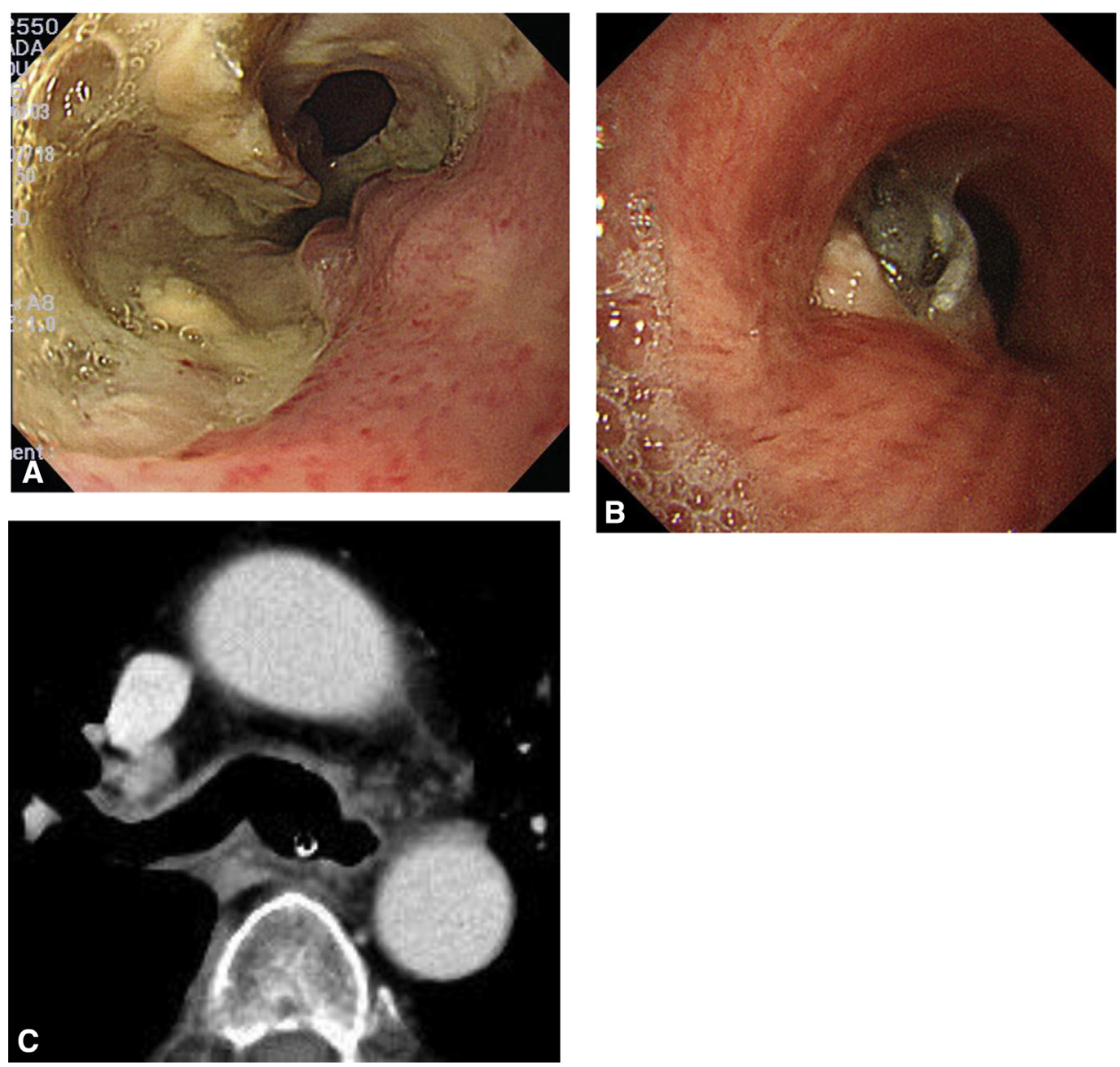

FIGURE 1. A, Upper gastrointestinal endoscopic image. B, Bronchoscopic image. C, Axial computed tomography image of the tracheoesophageal fistula.

associated with DCRT for ESCC. Without major necrosis of the esophageal wall or the tracheobronchial membranous portion, endotracheal stenting and/or esophageal bypass surgery are effective treatment strategies; however, in the present case with near-circumferential necrosis of the esophageal wall, surgically repairing the airway defect and preventing the development of an aortoesophageal fistula by means of esophagectomy was the only lifesaving option.

Grafts for patch repair generally comprise a free or pedicled pericardial patch $^{2-4}$ and muscle flaps from the latissimus dorsi, serratus anterior, or intercostal muscle. ${ }^{3,5}$ However, in post-DCRT cases, good blood perfusion of the graft is essential, given the protracted wound healing and increased susceptibility to infection due to decreased blood flow and severe scarring. Moreover, in cases with large airway defects, a patch graft with low compliance is required to resist the changes in intratracheal pressure and avoid airway stenosis or occlusion by endoluminal protrusion of the graft. In addition, a less invasive technique is one of the important requirements, considering the seriousness of the patient's condition. Thus, a pedicled pericardial patch is likely the best candidate, as a graft with adequate perfusion and low compliance that enables a less-invasive repair.

In respiratory surgery, a lateral pericardial flap is frequently harvested at the anterior mediastinum to reinforce the bronchial stump. ${ }^{3}$ However, when it comes to patch repair of the membranous portion of the tracheal bifurcation, the access route is longer, making tensionfree repair difficult. In contrast, a posterior pericardial flap is easily accessed after esophagectomy and allows patch repair via the shortest surgical route. In addition, harvesting and grafting of the flap can be performed in a sequence of surgical procedures. Moreover, in the present case, no repair of the posterior pericardial defect was needed, because the heart was unlikely to be displaced given that the thoracic vertebra covered the defect.

Intraoperative respiratory management is also an important problem. In our case, ECMO was used at the anesthesiologists' request; however, ECMO may have to be prepared only if a cuff of a double-lumen endotracheal tube can be placed in the LMB distal to the fistula, because oozing caused by heparinization increases blood loss and disturbs the surgical field of view. 

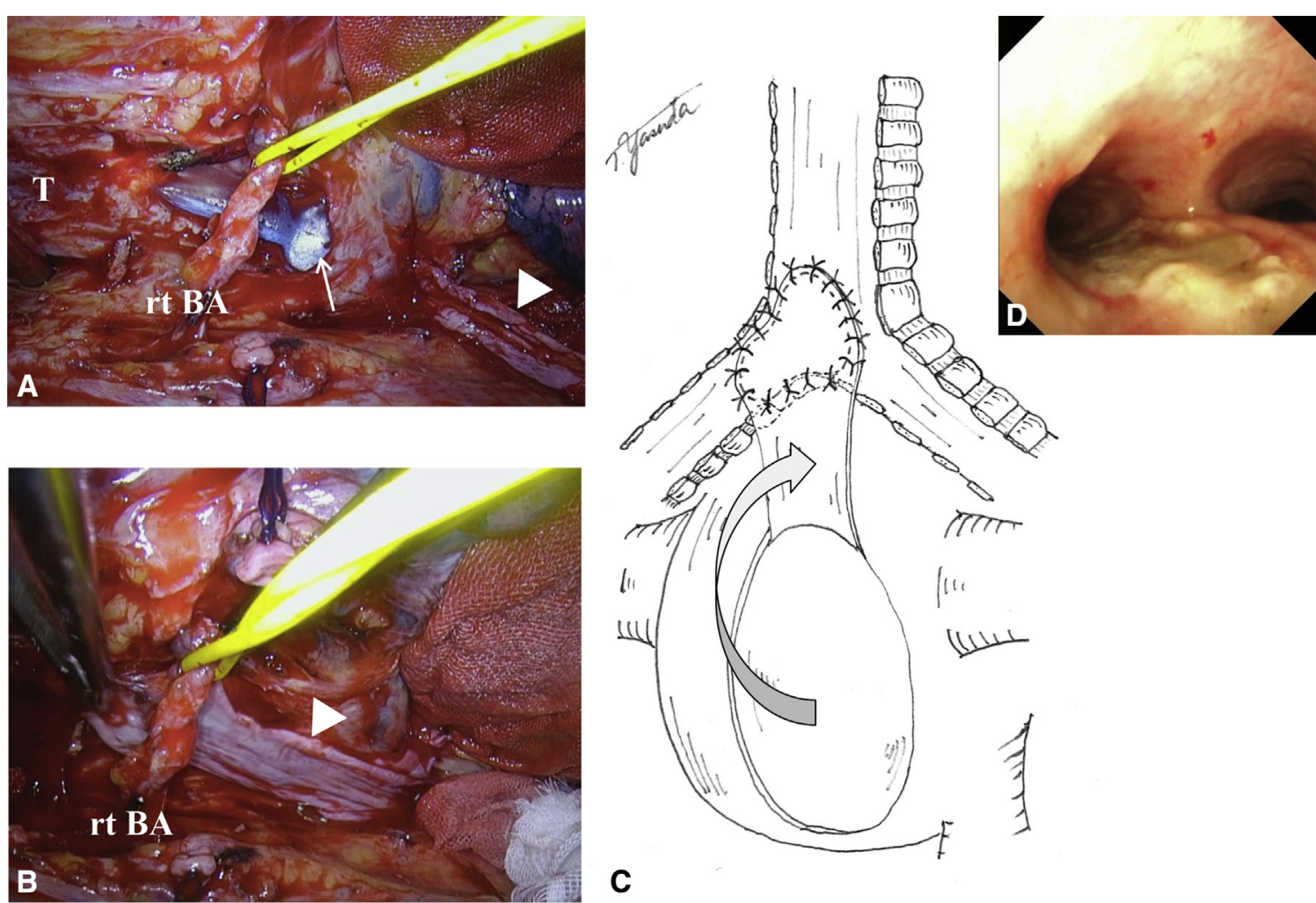

C

FIGURE 2. A, The fistula and bronchial cuff seen through the fistula (arrow). B, The pedicled posterior pericardium flap ( $\boldsymbol{\Delta})$ that was turned over and covered the defect (triangle). C, Schema of the pedicled posterior pericardial flap for repair of the airway defect. D, Postoperative bronchoscopic image of the pericardial patch. $T$, Trachea; $r t B A$, right bronchial artery.

DCRT is a useful modality for treating esophageal cancer at any stage; however, it is also associated with the risk of potentially fatal adverse events, such as a tracheoesophageal fistula, when the tumor is located in the middle to upper third of the esophagus. Our method using a pedicled posterior pericardial flap is a feasible option for such patients requiring surgical repair in the presence of severe scarring.

\section{References}

1. Gaspar LE, Winter K, Kocha WI, Coia LR, Herskovic A, Graham M. A phase I/II study of external beam radiation brachytherapy, and concurrent chemotherapy for patients with localized carcinoma of the esophagus. Radiation Therapy Oncology Group Study 9207 final report. Cancer. 2000;88:988-95.

2. Hagendoorn J, Schipper ME, Cloïn A, Ramjankhan FZ, Siersema PD, van Hillegersberg R. A patient with tracheoesophageal fistula and esophageal cancer after radiotherapy. Nat Rev Gastroentrol Hepatol. 2010;7:702-6.

3. Anderson TM, Miller JI. Use of pleura, azygos vein, pericardium, and muscle flaps in tracheobronchial surgery. Ann Thorac Surg. 1995;60:729-33.

4. Song S-W, Lee H-S, Kim MS, Lee JM, Kim JH, Zo JI. Repair of gastrotracheal fistula with a pedicled pericardial flap after Ivor Lewis esophagogastrectomy for esophageal cancer. J Thorac Cardiovasc Surg. 2006; 132:716-7.

5. Shen KR, Allen MS, Cassivi SD, Nichol FC III, Wigle DA, Harmsen WS, et al. Surgical management of acquired nonmalignant tracheoesophageal and bronchoesophageal fistulae. Ann Thorac Surg. 2010;90:914-9. 